\title{
Estrategia Experimental para la Visualización de Datos Urbanos: Visualización, Análisis y Evaluación de Flujos Vehiculares \& Transporte Público
}

\author{
Experimental Strategy for Urban Data Visualization: Visualization, Analysis and Evaluation of Vehicle \& \\ Transportation Flows
}

\author{
Alexis Salinas Arriagada \\ Universidad del Bio Bio, Chile. \\ asalinas@alumnos.ubiobio.cl
}

\begin{abstract}
The distance between the data and conventional graphical representation (line graphs, pie, bar), subtraction urban context information impoverishing their reading and understanding of territory, therefore his chances of inference, which leads to the omission of information for lack of representation. The urban spatial referencing such data could accommodate a much more thorough examination of these to a deeper realization of Analysis, Diagnostics and Evaluations for the city and its transport system is, as this research focuses his gaze, looking promote the efficient functioning of this important player in the city urban. This research presents the early development of a 'Strategy for representing experimental urban data "and visualization, using a graphical editor graphics algorithmic " Grasshopper ", as well as investigate their impact and how these can glimpse useful criteria to consider in the implementation and urban design.
\end{abstract}

Keywords: Urban data visualization; Experimental strategy; Streams \& transportation.

\section{Introducción}

"Las formas convencionales del urbanismo contemporáneo son concebidas desde un punto de vista espacial y temporal como "terminadas", mientras que este "nuevo urbanismo" intenta promover nuevas formas de ocupación del espacio, focalizándose en secuencias temporales de desarrollo y capacidades de crecimiento a largo plazo, permitiendo una adaptación y un cambio en los criterios de desarrollo y evolución dentro de la banda temporal en la que se ubica." (Polito, Castro, \& Ramos, 2009)

El estudio se sitúa en una zona inconexa de la ciudad, generada por la incidencia de las vías vehiculares y la vía férrea, con un lento progreso en términos urbanos, en un area que antecede al Río Bío Bío, territorio cuya directriz es hoy la de integrarse rio extendiendo al ciudad a él. Por lo que la oportunidad para plantear un nuevo urbanismo y junto con él, una nueva manera de entender la ciudad, es que se propone este campo como insumo de estudio.

Para la representación y entendimiento de la movilidad, el flujo y el transporte en la ciudad se plantean una serie 5 de ProtoMétodos tempranos;

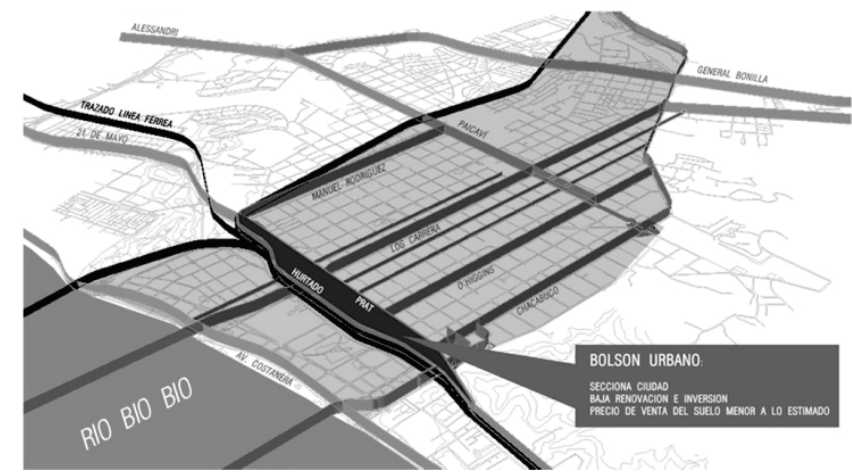

Figura 1: Situación Urbana, Ciudad de Concepción 


\section{Proto-Método 001:}

\section{Visualización origen/destino}

Plantea identificar cuales son los polos que atraen la mayor cantidad de personas a la ciudad y su origen, individualizarlos por tipo (salud, comercio, educacion etc). Se apunta a una mayor comprension y referenciacion espacial para este tipo de datos de insumo, buscando identificar las densidades de ubicación de estos polos y las zonas de vacios que ocurren en la ausencia de estos en la ciudad, ello permitirá evaluar la redistribucion futura de estos y una mejor utilizacion del territorio.

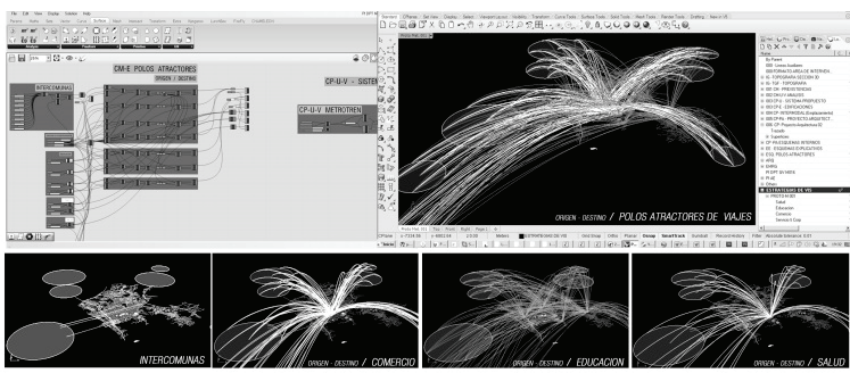

Figura 2: Proto-Método 001: Discriminando por polos de atracción de viajes, desde las intercomunas, educación, comercio, salud. (Cada fibra representara una unidad potenciada en 10 o 100, dependiendo de la densidad de la visualización y lo efectiva de esta dependiendo el caso)

\section{Proto-Método 002:}

\section{Identificación y valorización de flujos vehiculares}

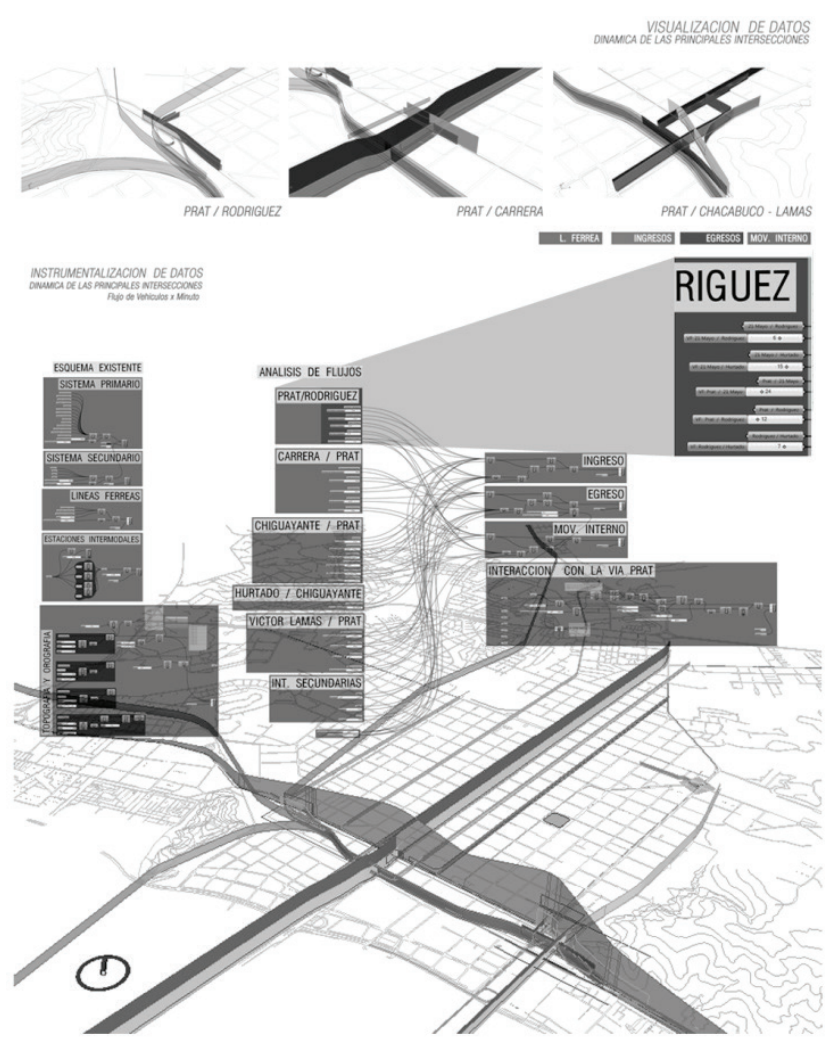

Figura 3: Proto-Método 002: Instrumentalización de datos, identificación de vías y valorización de flujos
La estrategia grafica los flujos vehiculares entendiendo; la jerarquía en ancho de las calles, defiendo así el sistema estructurante. Definido el escenario de flujos se toma como "sujeto" de estudio el tramo vial(es) desde donde las ciudad se percibe inconexa y se recogen datos de campo (Unidades vehiculares $\mathrm{X}$ Minuto $=\mathrm{VxM}$ ) en las intersecciones con las otras vías estructurantes, en horas punta; mañana, medio día y tarde, son ingresadas en el software paramétrico para ser graficadas, asignando estos datos como una variable para el eje Z, obteniendo un diagrama tridimensional donde su valor de flujo será visualizada mediante altura ( eje Z), obteniéndose con ello una visión integrada de la Información, que contrasta con el sistema tradicional de visualización en este contexto de estudio.

\section{Proto-Método 003:}

\section{Evaluador de Congestión}

Grafica mediante picos el nivel de congestión de una vía respecto otra perpendicular utilizando, como herramienta de Insumo, la cantidad de autos en espera al dar el rojo de semáforo. La existencia de semáforos en las Intersecciones, manifiesta una aglomeración de vehículos en ese lapso de tiempo dado, desencadenando vías y zonas de conflicto, traduciéndose en los llamados "Tacos". Tomando la reanudación de la circulación vial en "Vehículos x Minuto", se ingresa dato, esta vez para ser evaluado en su altura con la vía principal a la que intersecta, a la que se asignó un valor máximo arbitrario (como referencia), se traduce conceptualmente en el siguiente criterio de análisis. A mayor altura (mayor flujo $\mathrm{x}$ minuto) de las vías intersectantes respecto a la vía intersectada, arrojara por proximidad al valor máximo referencial, una gráfica que mostrara en los picos más altos las zonas de mayor conflicto vial "Tacos". Se permite aquí poseer una visualización general de incidencias, para posibilitar la toma decisión respecto a la redistribución de flujos y así homogeneizar la utilización de las vías, y descongestionar aquellas de mayor conflicto.

\section{Proto-Método 004:}

\section{Visualización de alimentación en una troncal}

Permite graficar una vía principal, troncal o estructurante respecto a las vías secundarias y como estas últimas alimentan el flujo de la primaria a medida que ingresan vehículos pasando a formar parte de torrente de circulación. La altura de cada banda refleja su tasa de vehículos por minuto, color anaranjado \& azul, corresponde al sentido de la alimentación del troncal, permitiendo visualizar desde que lugar del territorio es el mayor aporte a la red vial a evaluar. Lo graficado la banda centra, (color blanco), indica el comportamiento del troncal evidenciando, en los picos más altos, los puntos de ingreso de mayor aporte al flujo. Se busca con esta herramienta establecer, valorizar y categorizar vías de primer, segundo, de tercer, cuarto, quinto orden etc., de acuerdo a su aporte al torrente en la vía troncal dilucidando nuevas oportunidades de gestión y distribución, para optimización de las vías. 

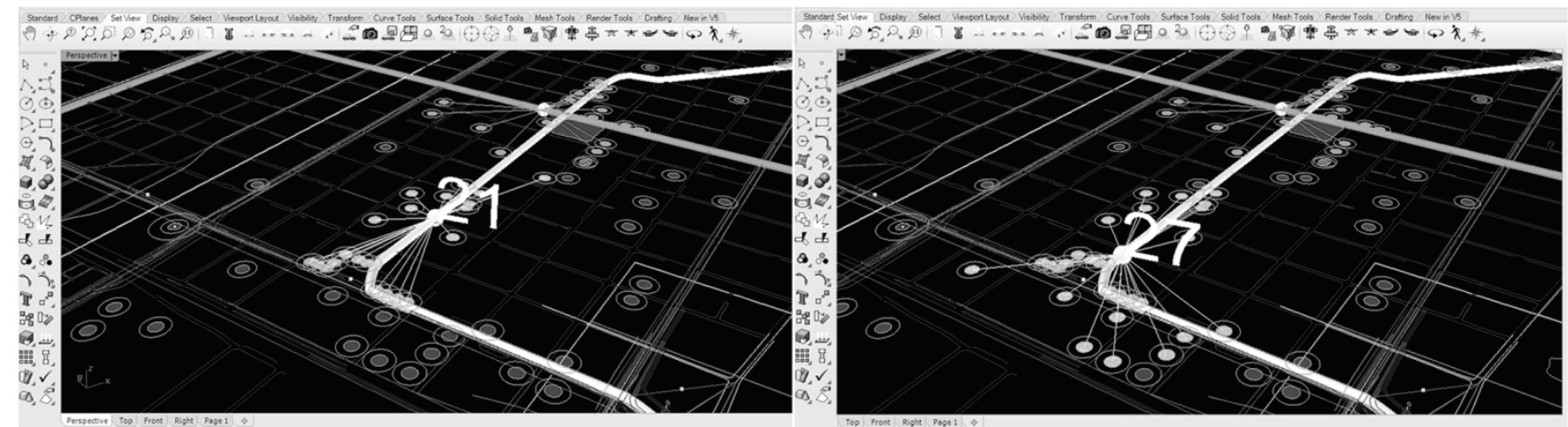

Figura 4: Proto-Método 003: Visualización, Grafica de Incidencias de flujos respecto a una vía Troncal.

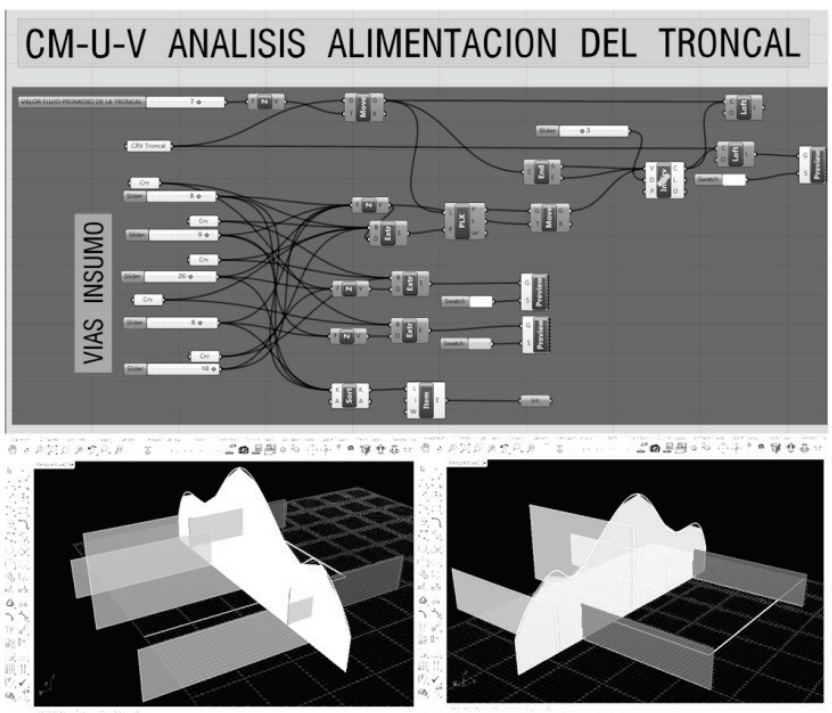

Figura 5: Proto-Método 004: Desarrollo conceptual, para la evaluación de una vía troncal.

\section{Proto-Método 005}

\section{Evaluador de proximidad para polos atractores}

Se plantea el escenario, para la toma de decisión respecto al recorrido de un Tranvía y sus lugares de detención dentro de la trama interna de la ciudad. Un punto, que simboliza el tranvía, en un recorrido dado, se evalúa y permitiendo establecer la máximas distancias de proximidad aceptable, en las que un usuario estuviese dispuesto a desplazarse para acceder al transporte público, $300 \mathrm{mts}$, (Observatorio Metropolitano, 2012) respecto a la mayor cantidad de polos atractores de destino insertos en la trama urbana de la ciudad posibilitando la visualización de los puntos de detención más favorables de acuerdo a este criterio, para decidir la ubicación de las detenciones de acuerdo a su mayor pertinencia en distancia e incidencia, situando los puntos de "parada" con un criterio de mayor eficiencia para transporte público.

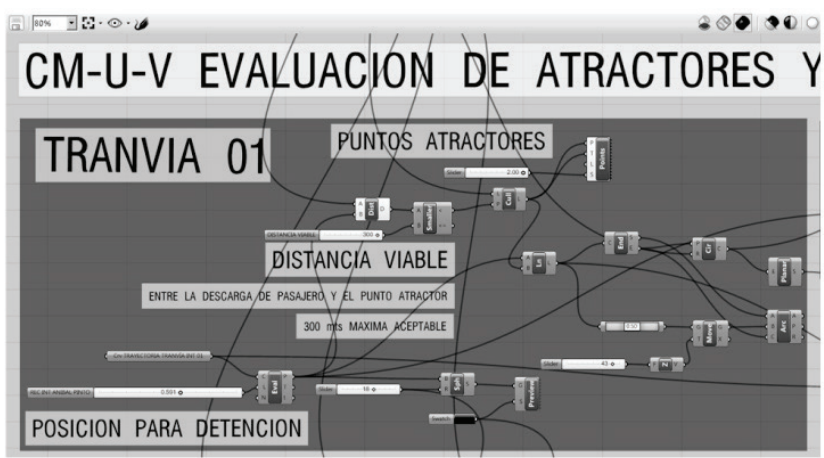

Figura 6:. Proto-Método 005: Detalle y extracto del algoritmo evaluador de proximidad.

El evaluador necesita poseer ya identificados los polos atractores de destino, los cuales se ingresan a un ítem común sin discriminar por, educación, comercio, salud, edificios y oficinas corporativas, servicios civiles etc. Permitiendo visualizar la cuantificación numérica de la totalidad de los polos incidentes en el punto de detención a evaluar.

\section{Conclusiones}

Comprendiendo la temprana etapa de desarrollo de esta investigación, pueden esbozarse conclusiones, que son respondidas con la propia visualización de los datos y la resultante de sus incidencias con un lenguaje fácilmente asimilable, otro de sus valores esta en la interpretación de los datos urbanos que al objetivizar percepciones de la ciudad, pudiesen gatillar decisiones y/o criterios de intervención para la Implementación, organización espacial, diseño y desarrollo de esta. La batería de "ProtoMétodos" expuesto en la investigación permite;

\section{Visualizar y comprender el territorio urbano.}

Entender comportamiento urbano respecto al transporte, utilización de vías, flujo e incidencias, además de en un futuro próximo potenciarse también como herramienta para la comprensión y estudio del flujo y el comportamiento peatonal. 


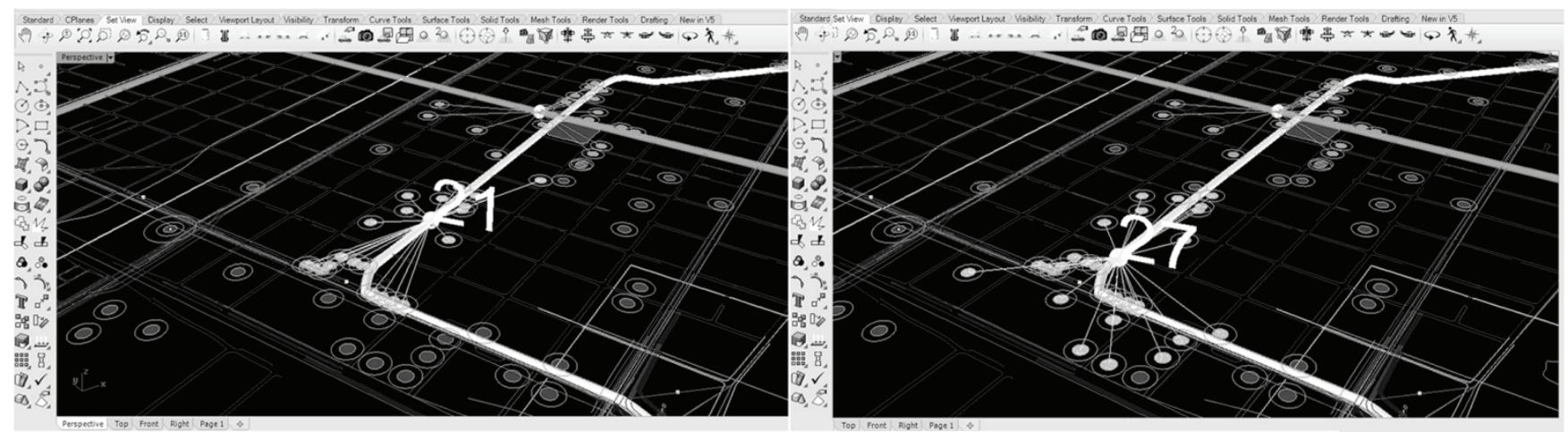

Figura 7: Proto-Método 005: Evaluación de recorrido y detención supuesto, para implementación de tranvía en las calles Prat / B. Aranas.

\section{Diagnosis y evaluación:}

Observar e inferir oportunidades, Incidir en la planificación, acción o intervención al mediano y/o largo plazo, en gestión para el comportamiento vehicular en la ciudad, pues permite la optimización en utilización de la red vial urbana y develar medidas que apunten hacia la eficiencia y la sustentabilidad del transporte en la ciudad.

\section{Toma de decisiones:}

Intervenir el espacio urbano, pues la información extraída de los análisis, permitirá la toma de decisiones respecto al uso del espacio público, permitiendo la reconfiguración del espacio urbano, como en el caso del evaluador de proximidad para la detención del transporte (Tranvía), que permitirá decidir la ubicación de la tal infraestructura, que afectará el espacio urbano existente.

La mayor potencialidad de la exploración de estas estrategias, está en la posibilidad de convertir a los "Proto-Métodos" en métodos y con ello en una herramienta más para el entendimiento de la ciudad y el manejo de las variables urbanas, no tan solo en ámbitos de flujos vehiculares, sino que también en los peatonales y en la integración de otras variables en las que la versatilidad del software de graficación algorítmica "Grasshopper", aún existen potencialidades inexploradas.

Insertar esta herramienta para el empleo en alguno de los distintos estamentos que estudian y planifican la Ciudad y el territorio, como lo pudiesen ser, Universidad, Planeamiento Regional y Departamentos Urbanos Municipales, son los campos de utilización en que se vislumbra la implementación al corto plazo, pues la complejidad actual en la ciudad exige nuevos campos de operación.

\section{Agradecimientos}

Al departamento de Posgrado de la Universidad del Bío Bío.

\section{Referencias}

Cáceres, k. (2011). Brechas intersticiales, Supuesto campo operativo. Universidad Tecnica Federico Santa Maria. Valparaiso, Chile.

Fernadez, M. (2011). http://www.ateneonaider.com/. Recuperado el 20 de Agosto de 2013, de http://www.ateneonaider.com/blog/manufernandez/10-ejemplos-de-visualizaci\%C3\%B3n-de-datos-urbanos

Ginebra, A. D. (2012). Ville Vivante. Recuperado el 16 de Septiembre de 2013, de http://villevivante.ch/: http://villevivante.ch/

Gonzales, M. C., Hidalgo, C. A., \& Barabasi, A.-L. (2013). Understanding Individual Human Mobility Patterns. Obtenido de http://www.nature.com:

http://www.nature.com/nature/journal/v453/n7196/full/nature0695 8.html

Observatorio Metropolitano. (2012). Primer Informe, Calidad de Vida Urbana del Gran Concepción. Concepción.

Polito, S., Castro, C., \& Ramos, E. (2009). Space, form, words. Recuperado el 15 de 03 de 2012, de Space, form, words: http://spaceformwords.wordpress.com/2009/05/20/ciudadinformal-urbanismo-parametrico-cocinando-flujos-de-informacion/ 